\title{
Detection of L-Proline-Catalyzed Michael Addition Reaction in Model Biomembrane
}

\author{
Masanori Hirose, Shigenori Sugisaki, Keishi Suga (D), and Hiroshi Umakoshi \\ Division of Chemical Engineering, Graduate School of Engineering Science, Osaka University, 1-3 Machikaneyama-cho, \\ Toyonaka, Osaka 560-8531, Japan \\ Correspondence should be addressed to Hiroshi Umakoshi; umakoshi@cheng.es.osaka-u.ac.jp
}

Received 3 October 2018; Revised 26 December 2018; Accepted 10 January 2019; Published 26 February 2019

Academic Editor: Josefina Pons

Copyright (C) 2019 Masanori Hirose et al. This is an open access article distributed under the Creative Commons Attribution License, which permits unrestricted use, distribution, and reproduction in any medium, provided the original work is properly cited.

\begin{abstract}
A method to detect the L-proline- (L-Pro-) catalyzed Michael addition reaction in model biomembranes has been established, using $N$-[ $p$ (2-benzimidazolyl)phenyl $]$ maleimide and acetone as reactants. The effect of liposome membranes on this reaction was kinetically analyzed using fluorescence spectroscopy. The kinetics of the reaction were different from those of the constituent lipids of the liposomes. Zwitterionic 1,2-dipalmitoyl-sn-glycero-3-phosphocholine liposome, which is in the solid-ordered phase, had a better value of reaction rate, suggesting that the reaction rate constants of this reaction in liposome membrane systems could be regulated by the characteristics of the liposome membrane (i.e., the phase state and surface charge). Based on the results obtained, a plausible model of the L-Pro-catalyzed Michael addition reaction was discussed. The obtained results provide us with an easily detectable method to assess the reactivity of L-Pro in biological systems.
\end{abstract}

\section{Introduction}

L-Proline (L-Pro) is one of the principle amino acids that are the building blocks of proteins. L-Pro is known to elucidate various kinds of physiological activities though its interference in the metabolic pathway, such as in the enhancement of growth of epidermal cells, activation of collagen biosynthesis, repair of damaged collagen, and moisture of the stratum corneum [1]. Considering these physiological activities, the use of L-Pro as a possible key material in food engineering has become significant.

As another significant aspect of L-Pro, it is gaining attention as an organocatalyst $[2,3]$. With respect to safety and high efficiency, the use of L-Pro will prove beneficial in industrial processes, such as in the manufacture of medicine and food. Barbas and coworkers have reported many examples of L-Pro-catalyzed reactions, especially focusing on enantioselectivity [4-6]. The L-Pro-catalyzed reaction is usually achieved through the formation of the "enamine" intermediate, between L-Pro and the ketone substrate. It is known that most of reported reactions have been carried out in the polar solvent, e.g., dimethyl sulfoxide (DMSO), but not in the high-polar solvent such as water, owing to the stability of the enamine intermediate. Considering the use of L-Pro in food engineering, it is thought that there could be some risks of the side reaction induced by L-Pro, as a less polar (hydrophobic) environment would exist in a biological system (e.g., in the interior of a biomembrane).

Recent studies have also focused on the use of selfassembly systems in organic synthesis because selfassemblies enable insoluble materials dispersed in aqueous media [7]. The liposome, a vesicular envelope composed of a phospholipid bilayer membrane, is known as a model of biomembranes. It can also be utilized as a platform of organic synthesis in aqueous media [8-11]. The liposome surface properties can be customized by modifying the lipid composition [12]. The combined use of small molecules with liposomes would lead to a cooperative alteration in the membrane properties, which can contribute to providing improved functions such as chiral recognition [13]. While the liposome structure has to be disrupted to recover the reaction product for its analysis, the use of the fluorescent 
reactant, for example, $N$-[ $p$ (2-benzimidazolyl)phenyl]maleimide (BIPM) [14], enables us to directly determine the reaction rate constant.

Previously, we have observed that L-amino acids, including L-Pro, can bind onto liposomal membranes [13]. Thereby, the L-Pro-catalyzed reactions can proceed on liposome membranes in aqueous media $[9,11]$. However, some challenges are still encountered in directly monitoring the kinetics of L-Pro-catalyzed reactions in the aqueous medium because the product is basically analyzed after extraction and purification. In this study, the L-Procatalyzed Michael addition reaction of BIPM and acetone was employed because BIPM is reported to be a good substrate to monitor L-Pro-catalyzed reactions through the fluorescence of the product [14]. The effect of liposome membranes on this reaction was kinetically analyzed using fluorescence spectroscopy. Based on the results obtained with previous findings, finally, a plausible model of the L-Pro-catalyzed Michael addition reaction was discussed. The obtained results provide an easily detectable method to assess the risk of L-Pro in biological systems.

\section{Materials and Methods}

2.1. Materials. 1,2-Dipalmitoyl-sn-glycero-3-phosphocholine (DPPC: C16:0, zwitterionic), 1,2-dioleoyl-sn-glycero-3phosphocholine (DOPC: C18:1, zwitterionic), and 1,2dipalmitoyl-3-trimethylammonium-propane (DPTAP: C16:0, cationic) were purchased from Avanti Polar Lipids, Inc. (Alabaster, AL, USA). L-Proline (L-Pro) and D-Proline were purchased from Peptide Institute (Osaka, Japan). $N$-[p(2-benzimidazolyl)phenyl]maleimide (BIPM) was purchased from Tokyo Chemical Industry co., Ltd. (Tokyo, Japan). Other chemicals were purchased from Wako Pure Chemical Industry Ltd. (Osaka, Japan) and were used as received.

\subsection{Liposome Preparation. Liposomes were prepared based} on literature [9]. In brief, a chloroform solution of lipids was dried in a round-bottom flask by using a rotary evaporator. The lipid film was dissolved in chloroform, and then the solvent was removed. The obtained lipid thin film was kept in a vacuum chamber for at least three hours, to remove the solvent completely. The dried lipid film was hydrated with ultrapure water at $60^{\circ} \mathrm{C}$. The liposome suspension was frozen at $-80^{\circ} \mathrm{C}$ for $15 \mathrm{~min}$ and was thawed at $60^{\circ} \mathrm{C}$ for $15 \mathrm{~min}$. This freeze-thaw cycle was repeated for five times. The liposome suspension was finally treated by using the extrusion device (Lipofast; Avestin Inc., Ottawa, Canada), with two layers of polycarbonate membranes with a mean pore diameter of $100 \mathrm{~nm}$. The size distributions of liposomes were determined by dynamic light scattering (DLS, Zetasizer Nano ZS, Malvern Panalytical, Grovewood Rd, UK). The average size of DPPC liposome just after preparation was $172.9 \mathrm{~nm}$ (PDI: 0.224 ). The average sizes of acetone added liposomes after 0 and $2 \mathrm{~h}$ were 143.3 and $247.3 \mathrm{~nm}$, respectively, revealing that the addition of acetone resulted in only small influences on the liposome structures.
2.3. Kinetics of L-Pro-Catalyzed Reaction in DMSO/Water System. L-Pro-catalyzed Michael addition of BIPM (BIPM: $28.9 \mu \mathrm{g}(0.1 \mu \mathrm{mol}))$ with acetone $(0.2 \mathrm{~mL}(2.7 \mathrm{mmol}))$ was conducted in DMSO or aqueous solution. L-Pro-catalyzed reaction was initiated by adding $10 \mu \mathrm{l}$ of stock solution of BIPM $(10 \mathrm{mM})$ in acetonitrile $\left(\mathrm{CH}_{3} \mathrm{CN}\right) / \mathrm{DMSO}(1 / 1 \mathrm{v} / \mathrm{v})$ mixture and $10 \mu \mathrm{l}$ of stock solution of L-Pro in water (0$8 \mathrm{mM})$ and acetone $(200 \mu \mathrm{l})$ to $780 \mu \mathrm{l}$ of DMSO or aqueous solution. The reaction was carried out in a quartz cell for measurement without stirring. The background fluorescence (before the reaction) was weak enough, and the increased fluorescence intensities were measured by the fluorescence spectrophotometer FP-8500 (JASCO, Tokyo, Japan) at $25^{\circ} \mathrm{C}$. The samples were excited with $315 \mathrm{~nm}$ and measured at $362 \mathrm{~nm}$. The reaction kinetic was considered by first-order kinetics, and the reaction rate constant, $k$, was determined by following equation:

$$
-\ln \left(1-\frac{\Delta I}{\Delta I_{\max }}\right)=k t,
$$

where $\Delta I$ and $\Delta I_{\max }$ represent the fluorescence at arbitral time $(t)$ and the final fluorescence intensity, respectively. The products were analyzed by HPLC (Waters 1500 HPLC System (Waters, Milford, MA, USA) equipped with the ODS2 column $(25 \mathrm{~cm})$ (GL Science Inc., Tokyo, Japan)). Mobile phase was prepared with $\mathrm{CH}_{3} \mathrm{CN} / 0.1 \%$ aqueous trifluoroacetic acid $(15: 85)$ at a flow rate of $1.0 \mathrm{~mL} / \mathrm{min}$, with detection at $310 \mathrm{~nm}$. The retention times of BIPM and product are 30.1 and $28.9 \mathrm{~min}$, respectively.

\subsection{L-Pro-Catalyzed Reaction in Liposome Membrane.} L-Pro-catalyzed reaction in the liposome membrane was initiated by adding $10 \mu \mathrm{l}$ of stock solution of BIPM $(5 \mathrm{mM})$ in $\mathrm{CH}_{3} \mathrm{CN} / \mathrm{DMSO}(1 / 1 \mathrm{v} / \mathrm{v})$ mixture and acetone $(200 \mu \mathrm{l})$ to a $790 \mu \mathrm{l}$ of mixture of liposome and L-Pro. A mixture of liposome and L-Pro was preincubated by mixing $780 \mu \mathrm{l}$ of the liposome membrane $(5 \mathrm{mM})$ and $10 \mu \mathrm{l}$ of L-Pro stock solution of water $(100 \mathrm{mM})$. The reaction was carried out in $5 \mathrm{ml}$ of the screw vial with stirring and light shielding. A sample for fluorescence measurement was prepared by diluting the reaction solution 50-fold with water. HPLC analyses were carried out as described above.

\section{Results and Discussion}

3.1. L-Pro-Catalyzed Reaction in DMSO and Water. BIPM is reported to be a good molecular probe to monitor the L-Pro-catalyzed reaction because its product shows fluorescence [14]. First, the L-Pro-catalyzed Michael addition reaction of BIPM and acetone (Scheme 1) was performed in DMSO. In the available literature, the reaction rate of the Michael addition reaction of BIPM and acetone, catalyzed by L-Pro, has been reported as $0.005 \mu \mathrm{M} / \mathrm{min}$ [14]. The 24-hour incubation period could be long enough to reach saturation in these reaction media. Figure 1 shows the fluorescence spectra of the products obtained in DMSO and water with an incubation period of 24 hours. The emission peak wavelength of the product could be dependent on the 


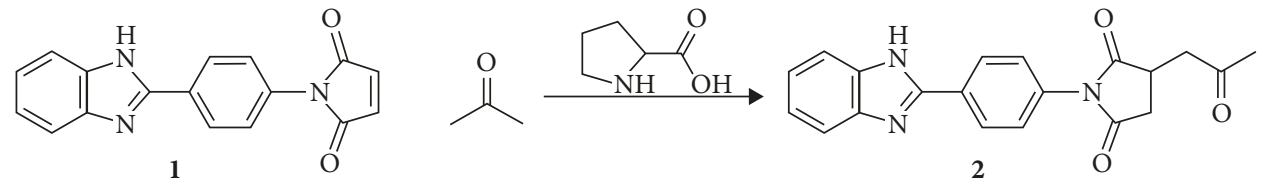

Scheme 1: Michael addition reaction of BIPM and acetone: (1) BIPM; (2) product.

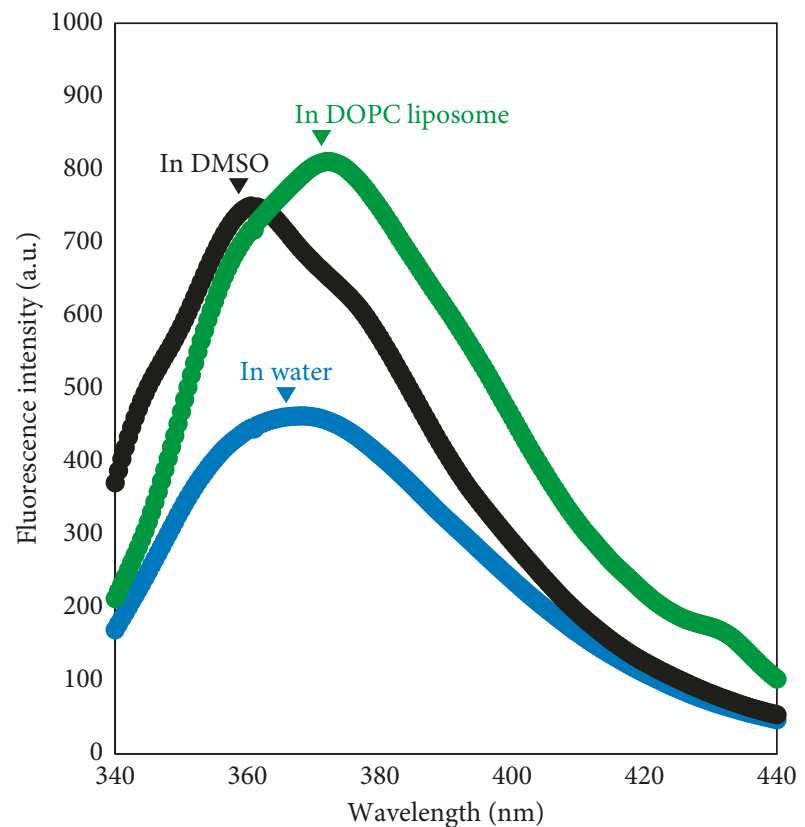

FIGURE 1: Fluorescence spectra of the products in DMSO, in water, and in DOPC liposome systems.

solvent; the wavelengths in DMSO and water were $360 \mathrm{~nm}$ and $368 \mathrm{~nm}$, respectively. In the 1,2-dioleoyl-sn-glycero-3phosphocholine (DOPC) liposome system, the emission peak was observed at $372 \mathrm{~nm}$ and the fluorescence intensity was slightly higher than that obtained in water. The fluorescent properties of the products are further discussed in Section 3.4. These results revealed that the product can emit strong fluorescence in these media.

In general, the L-Pro-catalyzed Michael reaction is faster in DMSO $[3,9]$. The dependence of amount of the catalyst in this reaction was then investigated by varying the amount of L-Pro. Figure 2(a) shows the time course of the fluorescence intensity at different concentrations of L-Pro in DMSO. The reaction rate constants $(k)$ were calculated based on first-order kinetics (equation (1)) (Figure 2(b)), suggesting that the reaction could proceed by following first-order kinetics, because the reaction rate and amount of L-Pro (catalyst) were almost in a linear relationship at L-Pro concentrations below $40 \mu \mathrm{M}$.

To investigate the effect of solvent polarity, the reaction was further conducted in the system of $\mathrm{DMSO} /$ water mixture. Figure 3 shows the time course of the fluorescence intensities, wherein the fluorescence intensities were monitored at $362 \mathrm{~nm}$. The presence of water significantly decreased the reaction rate. In general, the enamine formation can be inhibited by the solvent water [4]. It is suggested that water molecules also play an inhibitory role in this reaction. Common to the reaction mechanisms of the
L-Pro-catalyzed reactions, the formation of the enamine intermediate is the key to achieve efficiency and enantioselectivity [2, 3, 15], whereby the water molecules could decrease the stability of the enamine intermediate. During the formation of the enamine of L-Pro and acetone, unstable intermediates can be formed [16]. When the reaction is carried out in liposome membranes, such intermediates could be stabilized by interaction with phospholipid molecules [11]. These results clearly indicate that the efficiency of the L-Pro-catalyzed reaction of BIPM and acetone can be inhibited in an aqueous solution, owing to the lack of hydrophobic (dehydrated) environment. The reactions catalyzed by D-Proline (D-Pro) were also investigated, but the catalytic activity of D-Pro might be lower than that of L-Pro (Figure 3). In most cases, L-Pro and D-Pro show similar catalytic performances except for the enantioselectivity of the product [17]. However, in some examples, the yield of the product differed depending on the proline structure [18]. In the case of direct asymmetric aldol reaction between $p$ nitrobenzaldehyde ( $p \mathrm{NBA})$ and acetone, the D-Pro derivative drastically decreased the reaction yield [2]. The carboxyl group of L-Pro also produced a good yield [2]. It is assumed that L-Pro can improve the reactivity of the enamine intermediate, suggesting that the enamine composed by L-Pro (or its derivatives) shows higher reactivity as compared to D-Pro derivatives, but further determinations are needed to investigate their differences. In addition, as D-Pro is hardly adsorbed onto liposomes [13], it was difficult to perform the D-Pro-catalyzed reactions at the liposome membrane. In this study, the L-Pro-catalyzed Michael addition reactions of BIPM and acetone are further investigated in the following sections.

\subsection{Coexistence Effect of Liposome Membrane L-Pro-} Catalyzed Reaction in an Aqueous Solution. The coexistence effect of the liposome membrane on the L-Procatalyzed reaction of BIPM and acetone was investigated in water. Although L-Pro is a water-soluble molecule, it has been reported that L-Pro can be adsorbed onto the liposome surface by incubation for 48 hours [13]. After L-Pro was preincubated with the DPPC liposome for 24 or 48 hours, the Michael addition reaction was initiated by adding BIPM and acetone. Figure 4(a) shows the fluorescence spectra of the products at different incubation times. In the case of 24-hour preincubation, the spectra were not changed significantly, indicating that the reaction did not proceed. On the contrary, after 48-hour preincubation, the fluorescence intensities were drastically increased, showing that the L-Pro-catalyzed reaction of BIPM and acetone was achieved in the aqueous medium when the liposome was coexisting. The adsorption 


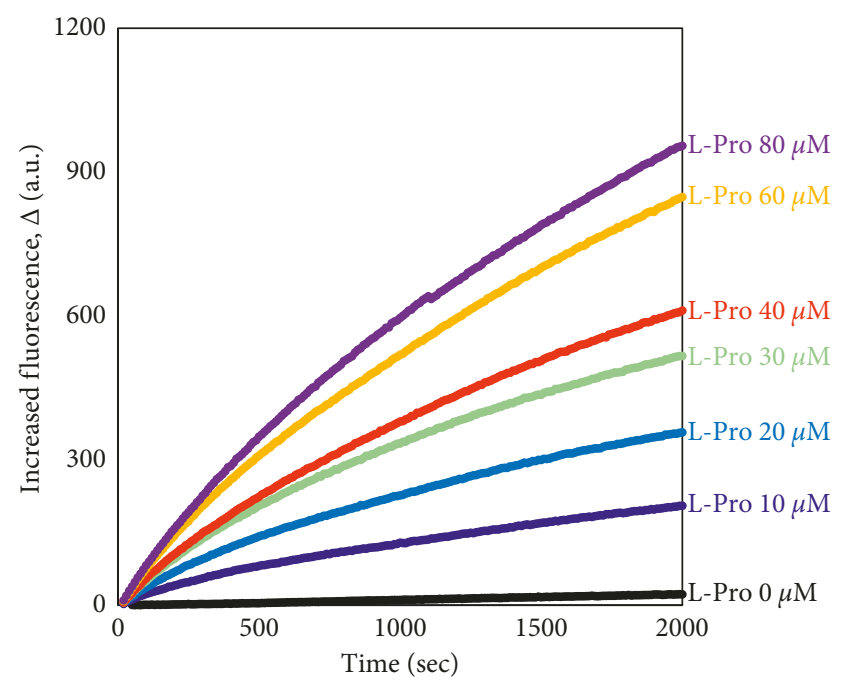

(a)

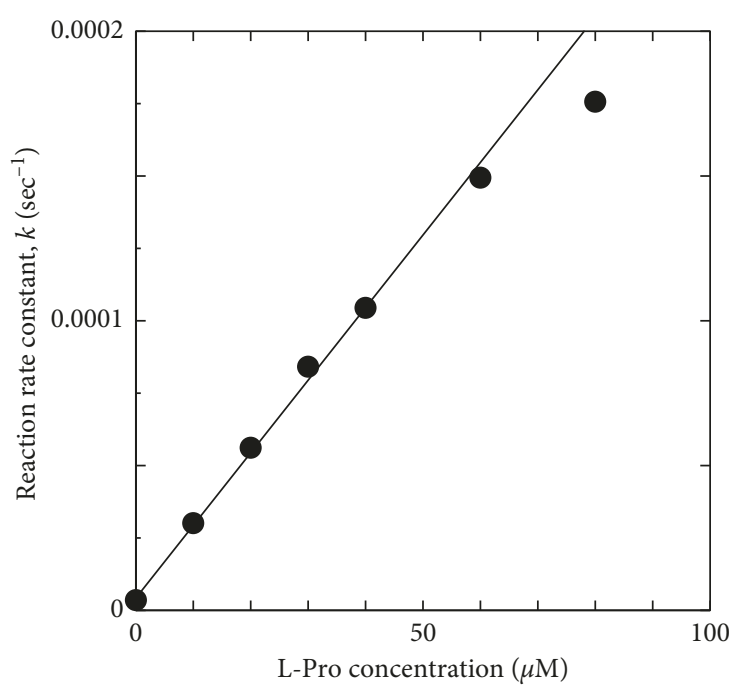

(b)

Figure 2: (a) Time course of the fluorescent intensity from the reaction product, at different L-Pro concentrations. (b) Relationship between L-Pro concentration and reaction rate constant.

amounts of L-Pro onto liposomes were approximately $10 \%$ of the total L-Pro- $8.3 \%$ for the DOPC liposome, $10.8 \%$ for the DPPC liposome, and $11.4 \%$ for the DPPC/DPTAP liposome, at 48 -hour incubation. We assume that only the adsorbed L-Pro possesses high catalytic activity, whereas the unadsorbed L-Pro can behave as free L-Pro. Although we have not estimated the L-Pro adsorption amount at 24 hours, in almost all cases, the adsorption amount at 24 hours is less than $20 \%$ of the maximum adsorption amount (at 48 hours). Considering these results and findings, the adsorption amounts of L-Pro could be estimated as $<100 \mu \mathrm{M}$ for 48 hour preincubation and $<20 \mu \mathrm{M}$ for 24 -hour preincubation. This could be the reason why the reaction rate constants in liposome systems turn out to be lower than those in the DMSO solvent.

Figure 4(b) shows the time course of the fluorescence intensity, showing that the $k$ value at 48 -hour preincubation was five times higher than that of 24 hour preincubation. It has been reported that the L-amino acid adsorption did not proceed until 24 hours and then dramatically proceeded within 24 - to 48 hour preincubation [13], in which the liposome surface properties could be varied by the adsorption of L-amino acids and then concerted binding could be initiated. The obtained results show that the Michael addition reaction of BIPM and acetone was promoted by the L-Pro that was adsorbed onto the membrane surface. Considering the facts that (1) water molecules principally inhibit the L-Pro-catalyzed reaction and (2) the liposome membrane provides a hydrophobic environment in the aqueous medium, the L-Pro-catalyzed reactions can be carried out in an aqueous medium, owing to the presence of the liposome membranes. The potential roles of the liposome membrane are to carry the hydrophobic reactant (BIPM) in the interior region of the membrane and to accumulate reactants and a catalyst in the dehydrated environment.

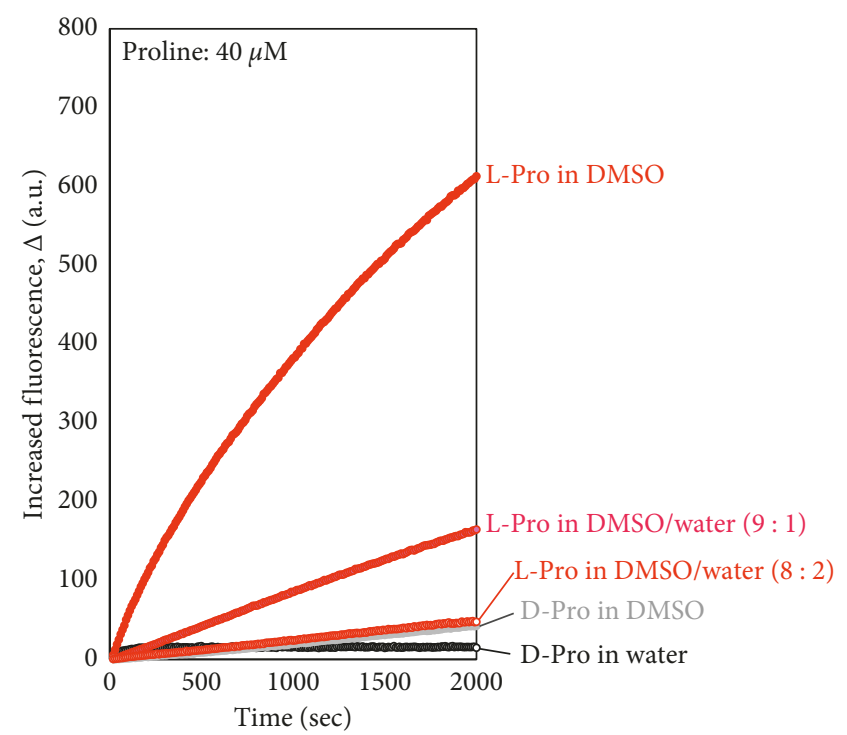

Figure 3: L-Pro-catalyzed Michael addition reaction of BIPM and acetone in DMSO/water solutions.

3.3. Effect of Type of Liposome Membrane on the L-ProCatalyzed Reaction. Biological membranes consist of various kinds of lipids and proteins. Among the naturally occurring zwitterionic phospholipids, DOPC and 1,2-dipalmitoyl-snglycero-3-phosphocholine (DPPC) were selected to investigate the effect of membrane properties on this reaction. At room temperature, DOPC and DPPC liposomes are in the fluid state (liquid-disordered, $\mathrm{L}_{\mathrm{d}}$, phase) and rigid state (solid-ordered, $s_{\mathrm{o}}$, phase), respectively. It is also reported that the cationic liposome DPPC/DPTAP (1,2-dipalmitoyl-3-trimethylammoniumpropane) can improve the L-Pro-catalyzed Michael addition reaction [9]. After L-Pro preincubation for 48 hours, the reaction of BIPM and acetone was carried out in the presence of the abovementioned liposomes (Figure 5). The initial reaction 


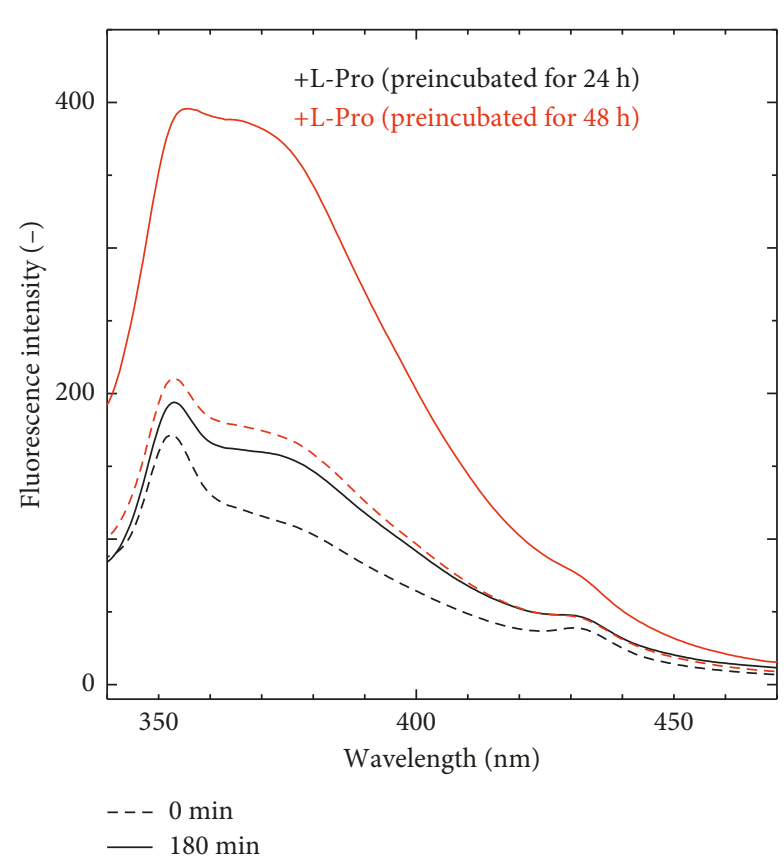

(a)

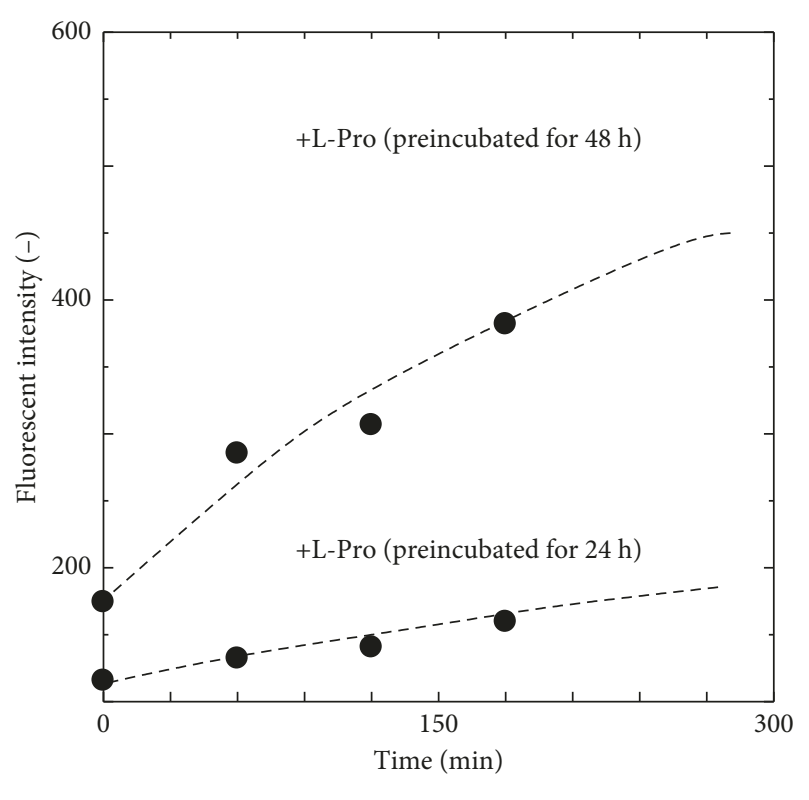

(b)

FIGURE 4: Coexistence effect of DPPC liposomes on L-Pro-catalyzed Michael addition reaction of BIPM and acetone: (a) fluorescence spectra of products; (b) time course of fluorescence emission peaks at $370 \mathrm{~nm}$. L-Pro was preincubated with DPPC liposome for 24 hours and 48 hours, before initiation of the reaction.

rate was herein analyzed by the slope of the approximately straight line of the plot of fluorescence intensity at $372 \mathrm{~nm}$ against time, as the fluorescence intensity increased proportionally with time. The initial reaction rate in DPPC ( $s_{\mathrm{o}}$ phase) was 2.5 times higher than that in DOPC ( $\mathrm{L}_{\mathrm{d}}$ phase). Furthermore, the addition of cationic DPTAP decreased the initial reaction rate, although both the DPPC and DPPC/DPTAP liposomes are in rigid membrane states ( $s_{\text {o }}$ phases) [9].

The membrane surface of the DPPC liposome is known to be in a dehydrated state as compared to that of the DOPC membrane [9], which could be favorable to stabilize the enamine intermediate of L-Pro and acetone. In addition, the "ordered" state of the DPPC membrane could contribute to promote the adsorption of the L-amino acid (including L-Pro), whereas L-amino acid adsorption can decrease when the membrane is in disordered states [13]. The adsorption of L-Pro was slightly increased by the positively charged liposomes [9], whereas the membrane property differences between DPPC and DPPC/DPTAP could be negligible. Thus, the inhibitory effect of the DPTAP on the reaction could be because of the positive charge of the membrane. Furthermore, only the positively charged molecules (e.g., propranolol) adsorbed onto the negatively charged liposomes [19]. It is assumed that L-Pro could preferentially adsorb onto the membrane in the $s_{\mathrm{o}}$ phase, in which the less hydrophobic environment contributes both to the adsorption of L-Pro and to the promotion of the Michael addition reaction.

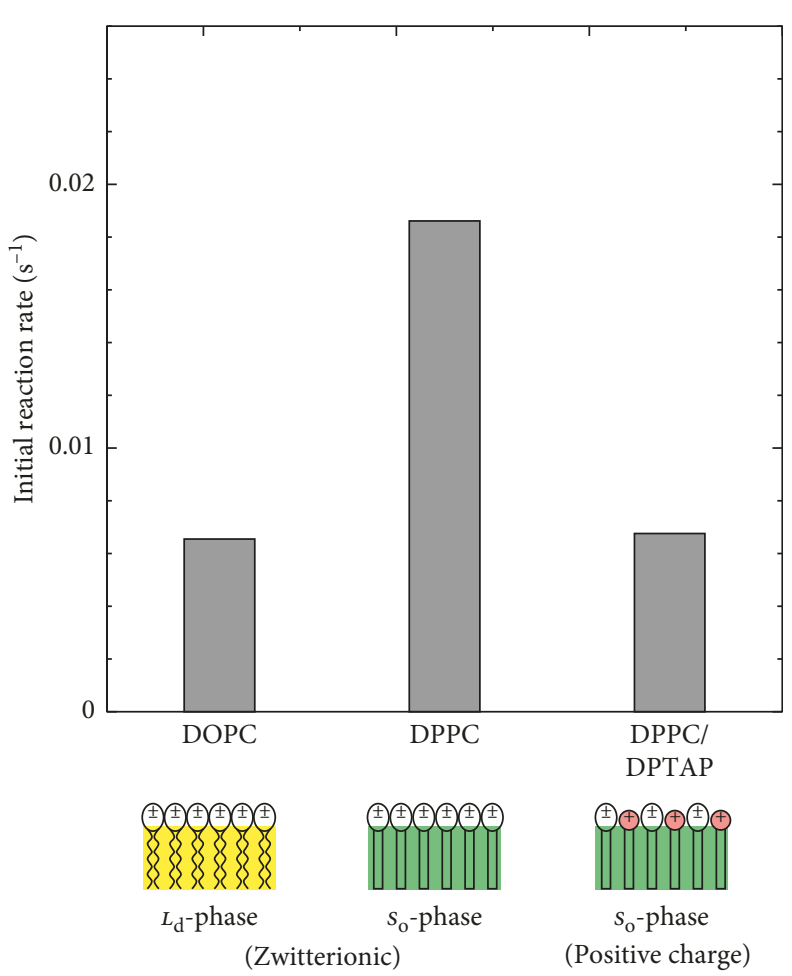

Figure 5: Comparison of the liposome phase state and initial reaction rate.

3.4. Plausible Model of L-Pro-Catalyzed Michael Addition Reaction of BIPM and Acetone in Liposome Membrane. Based on the abovementioned results, a plausible model of the L-Pro-catalyzed Michael addition reaction is discussed. To 


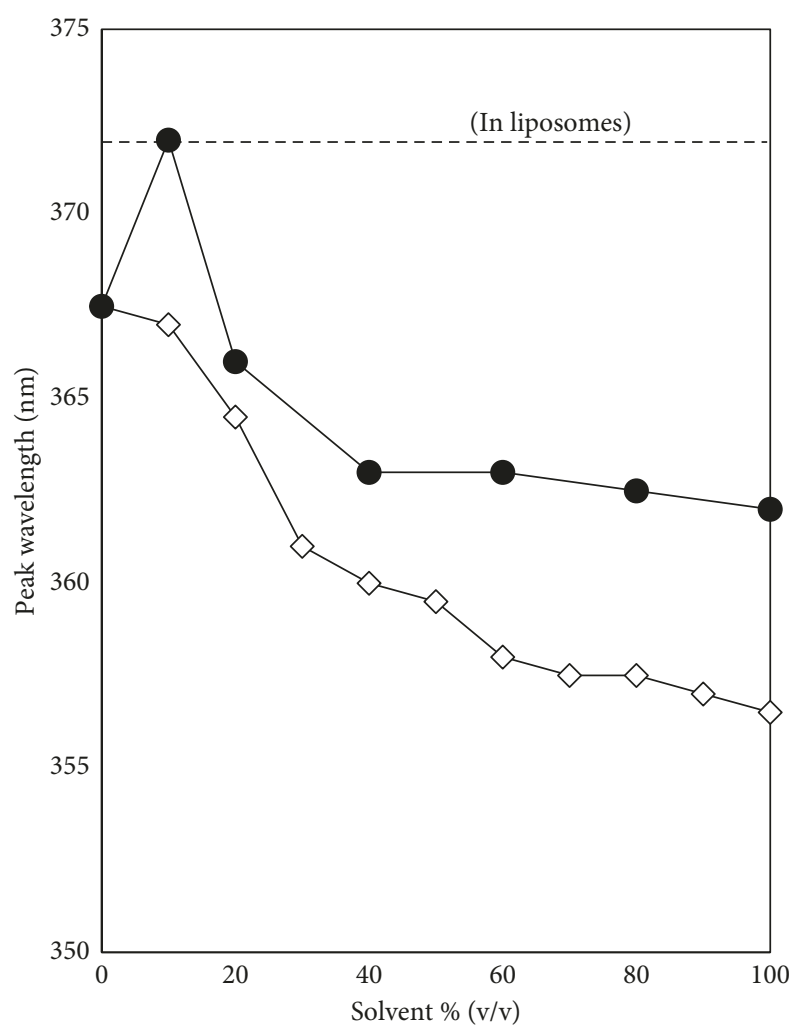

(a)

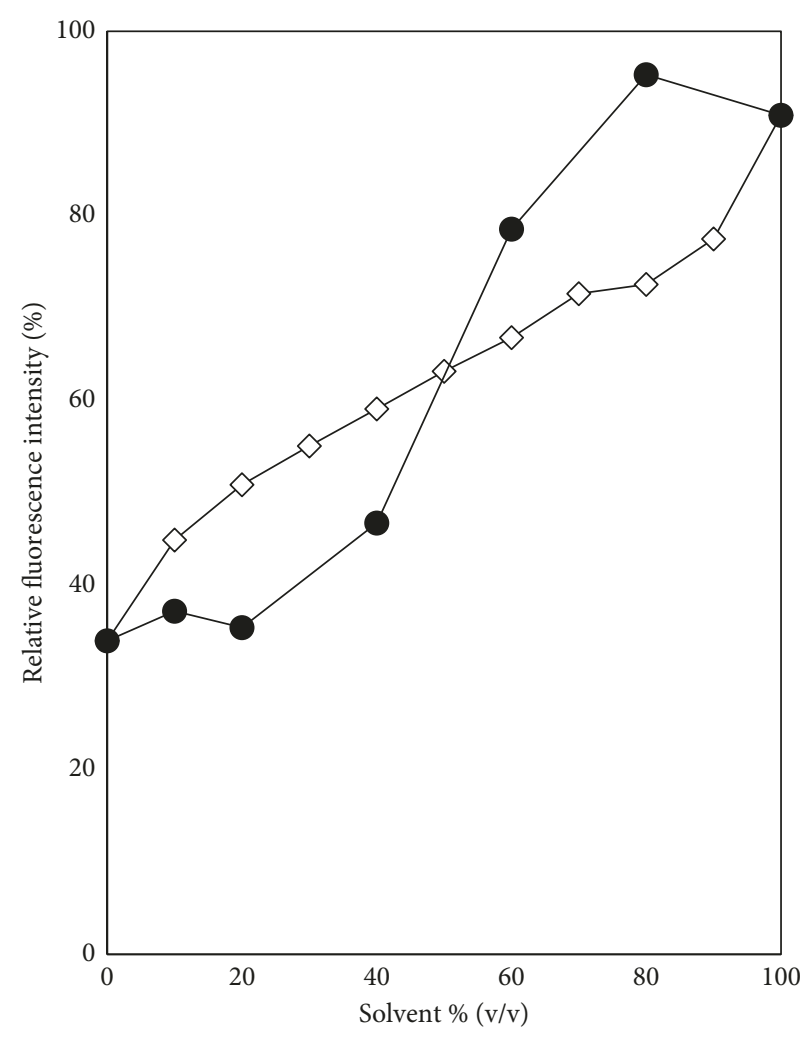

(b)

FIGURE 6: Emission peak wavelength (a) and relative emission peak intensity (b) of the reaction products in 1,4-dioxane/water mixtures (open diamond) and in DMSO/water mixtures (closed circle).

assess the location of the product in the liposome membrane, the peak shift of the emission fluorescence of the reaction product was analyzed in various kinds of solvents with different polarities. In the existing literature, it has been reported that the fluorescence intensity of the product in DMSO was slightly higher ( 1.6 times) than that in water [14]. To verify the solvatochromism of the product, the product recovered in DMSO solvents after 24 hour incubation was transferred into $\mathrm{DMSO} /$ water mixtures for further analysis of its fluorescence. Figure 6(a) shows the emission peak wavelength of the reaction product in both 1,4-dioxane/water systems and $\mathrm{DMSO} /$ water systems. The emission peaks gradually redshifted as the water ratio increased. In the liposome systems, the emission peaks were observed at $368.5 \mathrm{~nm}$ (we confirmed the emission peaks with three isolated data from DOPC and DPPC). This suggests that the products generated in the liposome tend to locate at the hydrophilic part (near the lipid head groups) although the emission peaks were broader in the liposome systems. The emission peak intensities were decreased in proportion to the water ratio (Figure 6(b)). The fluorescence intensity of the product in water decreased to $40 \%$. In contrast, the presence of water in the reaction mixture drastically inhibited the reaction (Figure 3), suggesting that the formation of the enamine intermediate of L-Pro and acetone could be the key to promote this reaction. The DMSO environment is suitable to stabilize enamine $[2,3]$, and a similar effect can be obtained by utilizing the liposome membrane $[9,11]$.

The possible locations of the molecules related to this reaction are summarized in Figure 7. L-Pro, the enamine intermediate, and acetone could be basically localized at the interface region, near the phosphate and glycerol groups; therefore, the enamine intermediate could be stably trapped there. The reaction could be completed by the attack of the enamine intermediate to BIPM, which could be located in a more hydrophobic region-near the hydrocarbon chains of lipids. Similarly, the reactants trans$\beta$-nitrostyrene ( $t \beta \mathrm{NS})$ and $p \mathrm{NBA}$ can be estimated to be located at the hydrophobic site $[9,11]$. The obtained results and previous findings suggest that the enamine intermediate, composed of L-Pro and acetone, could preferentially locate in the hydrophobic region of the liposome membrane, which successfully promotes the L-Pro-catalyzed reactions. The efficiency and enantioselectivity can be varied, preferably depending on the property of the reactant $-t \beta$ NS would provide high enantioselectivity; $p$ NBA would provide high conversion but low enantioselectivity. For BIPM, although the conversion was lower, the reaction kinetics was directly analyzed in the aqueous medium, revealing that the L-Procatalyzed Michael addition of BIPM and acetone was surely performed in the liposomal membrane. 


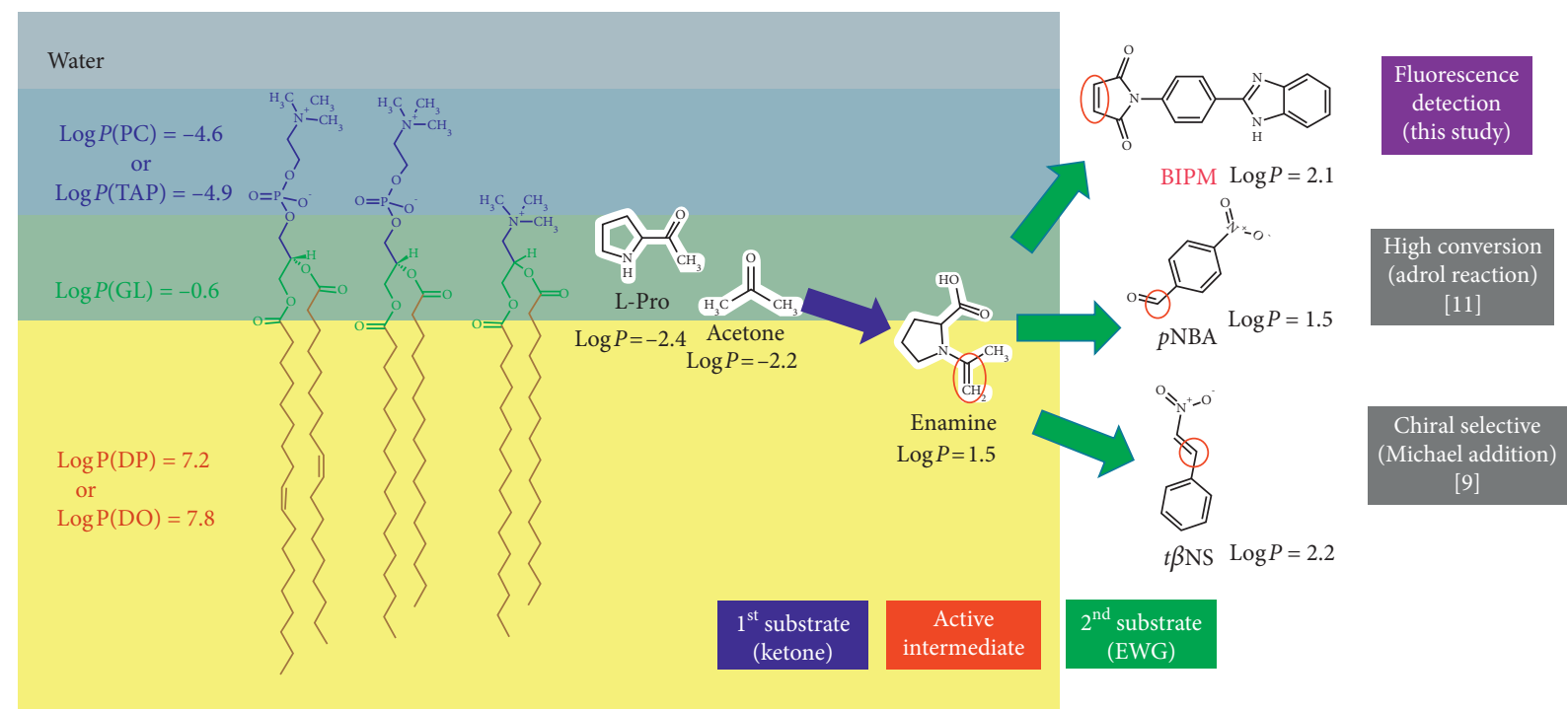

Figure 7: Schematic illustration of the location of the molecules related to the L-Pro-catalyzed Michael addition reaction in liposome membranes.

\section{Conclusions}

The kinetics of the L-Pro-catalyzed Michael addition reaction of BIPM and acetone were analyzed by using fluorescence spectroscopy. The composition of liposomes could be a controlling factor in this reaction-the zwitterionic DPPC liposome displayed the highest reaction rate constant among the liposomes tested in this work. From the point of view of green chemistry, organic synthesis in aqueous media provides various benefits. On the contrary, in food science and engineering fields, our findings provide a warning that the L-Pro distributed into biomembranes (hydrophobic environment) can react with ketones, which might lead to unexpected and inconvenient chemical reactions in the human body. The ketones can be produced by lipid oxidation in biomembranes; thus, ketones in biomembranes would be reactive in the presence of L-Pro, which might result in unexpected products. Thus, in situ detection of the L-Pro-catalyzed reactions is necessary. The obtained results provide us with an easily detectable method to assess the risk of L-Pro in biological systems, which can be detected using BIPM as a donor reactant in the L-Pro-catalyzed Michael addition reaction.

\section{Abbreviations}

L-Pro: L-Proline

DMSO: Dimethyl sulfoxide

BIPM: $\quad N$-[p(2-Benzimidazolyl $)$ phenyl $]$ maleimide

DPPC: 1,2-Dipalmitoyl-sn-glycero-3-phosphocholine

DOPC: 1,2-Dioleoyl-sn-glycero-3-phosphocholine

DPTAP: 1,2-Dipalmitoyl-3-trimethylammonium-propane

$T_{\mathrm{m}}: \quad$ Phase transition temperature

MLV: Multilamellar vesicle

LUV: Large unilamellar vesicle

$\mathrm{CH}_{3} \mathrm{CN}$ : Acetonitrile

D-Pro: D-Proline

pNBA: $\quad p$-Nitrobenzaldehyde
$\mathrm{L}_{\mathrm{d}}: \quad$ Liquid disordered

$s_{\mathrm{o}}$ : $\quad$ Solid ordered

$t \beta \mathrm{NS}: \quad$ trans- $\beta$-Nitrostyrene.

\section{Data Availability}

The data used to support the findings of this study are included within the article.

\section{Conflicts of Interest}

The authors declare that there are no conflicts of interest regarding the publication of this paper.

\section{Acknowledgments}

This work was supported by the JSPS Grant-in-Aid for Scientific Research A (26249116), Challenging Exploratory Research (T15K142040), and Sasakawa Scientific Research Grant from the Japan Science Society (29-336). We thank Dr. Takaaki Ishigami and Dr. Yukihiro Okamoto (Graduate School of Engineering Science, Osaka University) for their constructive comments and technical support.

\section{References}

[1] P. Li and G. Wu, "Roles of dietary glycine, proline, and hydroxyproline in collagen synthesis and animal growth," Amino Acids, vol. 50, no. 1, pp. 29-38, 2017.

[2] B. List, R. A. Lerner, and C. F. Barbas, "Proline-catalyzed direct asymmetric aldol reactions," Journal of American Chemical Society, vol. 122, no. 10, pp. 2395-2396, 2000.

[3] B. List, P. Pojarliev, and H. J. Martin, "Efficient prolinecatalyzed Michael additions of unmodified ketones to nitro olefins," Organic Letters, vol. 3, no. 16, pp. 2423-2425, 2001.

[4] N. Mase, K. Watanabe, H. Yoda, K. Takabe, F. Tanaka, and C. F. Barbas, "Organocatalytic direct Michael reaction of ketones and aldehydes with $\beta$-nitrostyrene in brine," Journal 
of American Chemical Society, vol. 128, no. 15, pp. 4966-4967, 2006.

[5] K. Sakthivel, W. Notz, T. Bui, and C. F. Barbas, "Amino acid catalyzed direct asymmetric aldol reactions: a bioorganic approach to catalytic asymmetric carbon-carbon bondforming reactions," Journal of the American Chemical Society, vol. 123, no. 22, pp. 5260-5267, 2001.

[6] T. Bui and C. F. Barbas, "A proline-catalyzed asymmetric Robinson annulation reaction," Tetrahedron Letters, vol. 41, no. 36, pp. 6951-6954, 2000.

[7] A. E. C. Palmqvist, "Synthesis of ordered mesoporous materials using surfactant liquid crystals or micellar solutions," Current Opinion in Colloid \& Interface Science, vol. 8, no. 2, pp. 145-155, 2003.

[8] F. Iwasaki, K. Suga, and H. Umakoshi, "Pseudo-interphase of liposome promotes 1,3-dipolar cycloaddition reaction of benzonitrile oxide and $\mathrm{N}$-ethylmaleimide in aqueous solution," Journal of Physical Chemistry B, vol. 119, no. 30, pp. 9772-9779, 2015.

[9] M. Hirose, T. Ishigami, K. Suga, and H. Umakoshi, "Liposome membrane as a platform for the 1-pro-catalyzed Michael addition of trans- $\beta$-Nitrostyrene and acetone," Langmuir, vol. 31, no. 47, pp. 12968-12974, 2015.

[10] F. Iwasaki, K. Suga, Y. Okamoto, and H. Umakoshi, "Liposomes can achieve enantioselective $\mathrm{C}-\mathrm{C}$ bond formation of an a-amino acid derivative in aqueous media," ACS Omega, vol. 2, no. 1, pp. 91-97, 2017.

[11] M. Hirose, S. Fujiwara, T. Ishigami, K. Suga, Y. Okamoto, and H. Umakoshi, "Liposome membranes assist the L-proline-catalyzed aldol reaction of acetone and p-nitrobenzaldehyde in water," Chemistry Letters, vol. 47, no. 7, pp. 931-934, 2018.

[12] K. Suga, K. Akizaki, and H. Umakoshi, "Quantitative monitoring of microphase separation behaviors in cationic liposomes using HHC, DPH, and laurdan: estimation of the local electrostatic potentials in microdomains," Langmuir, vol. 32, no. 15 , pp. 3630-3636, 2016.

[13] T. Ishigami, K. Suga, and H. Umakoshi, "Chiral recognition of 1-amino acids on liposomes prepared with 1-phospholipid," ACS Applied Materials \& Interfaces, vol. 7, no. 38, pp. 21065-21072, 2015.

[14] F. Tanaka, R. Thayumanavan, and C. F. Barbas, "Fluorescent detection of carbon-carbon bond formation," Journal of American Chemical Society, vol. 125, pp. 8523-8528, 2003.

[15] B. List, P. Pojarliev, W. T. Biller, and H. J. Martin, "The proline-catalyzed direct asymmetric three-component mannich reaction: scope, optimization, and application to the highly enantioselective synthesis of 1,2-amino alcohols," Journal of American Chemical Society, vol. 124, no. 5, pp. 827-833, 2002.

[16] U. I. Tafida, A. Uzairu, and S. E. Abechi, "Mechanism and rate constant of proline-catalysed asymmetric aldol reaction of acetone and p-nitrobenzaldehyde in solution medium: density-functional theory computation," Journal of Advanced Research, vol. 12, pp. 11-19, 2018.

[17] D. Mandalapu, "L-proline and d-proline (chiral amino acid catalysts)," Synlett, vol. 26, no. 5, pp. 707-708, 2015.

[18] E. Conde, D. Bello, A. de Cózar, M. Sánchez, M. A. Vázquez, and F. P. Cossío, "Densely substituted unnatural 1- and d-prolines as catalysts for highly enantioselective stereodivergent $(3+2)$ cycloadditions and aldol reactions," Chemical Science, vol. 3, no. 5, pp. 1486-1491, 2012.

[19] T. Ishigami, M. S. Chern, K. Suga, Y. Okamoto, and H. Umakoshi, "Adsorption behavior of propranolol on negatively-charged liposomes and its influence on membrane fluidity and polarity," Journal of Nanoscience and Nanotechnology, vol. 17, no. 3, pp. 1721-1728, 2017. 

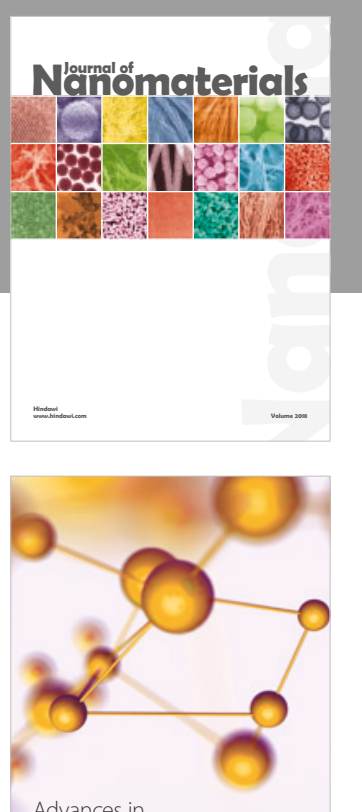

Physical Chemistry
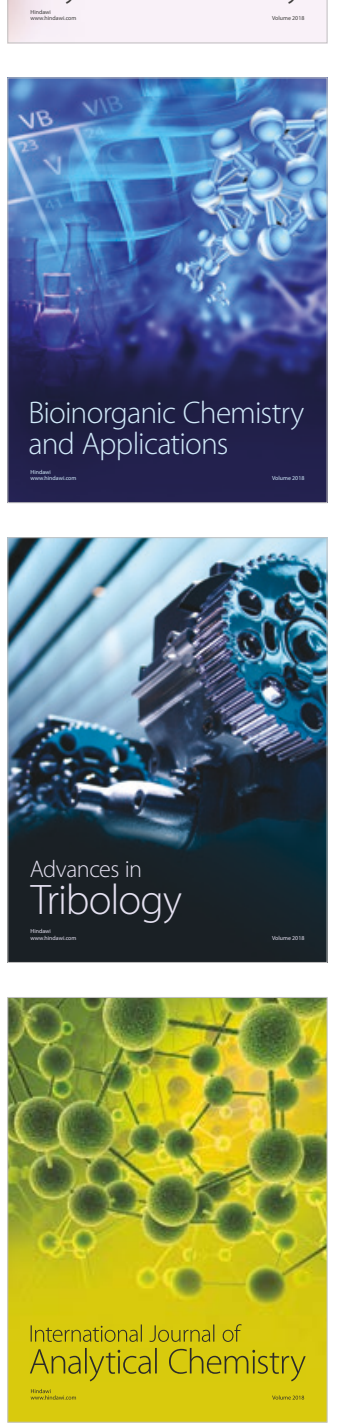

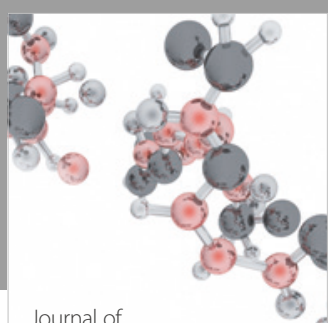

Analytical Methods

in Chemistry

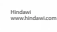

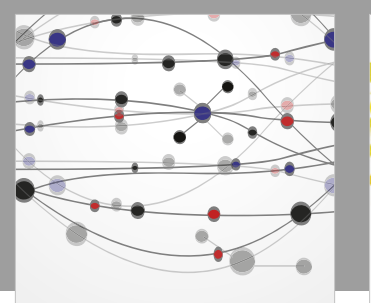

The Scientific World Journal

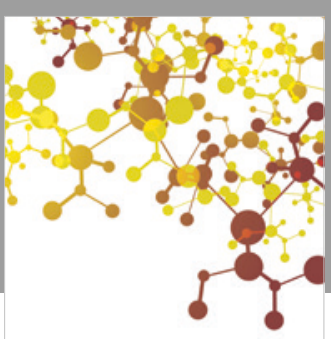

Journal of

Applied Chemistry
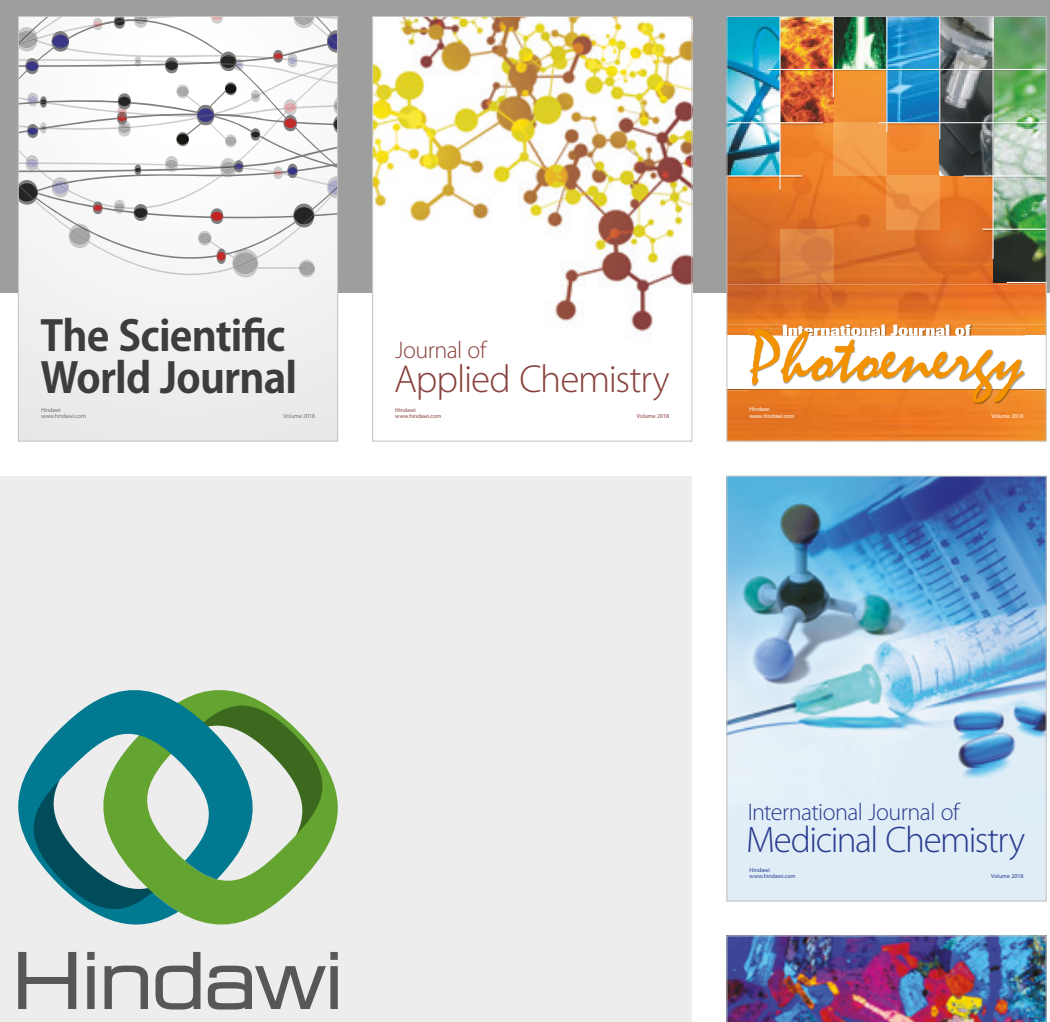

Submit your manuscripts at

www.hindawi.com
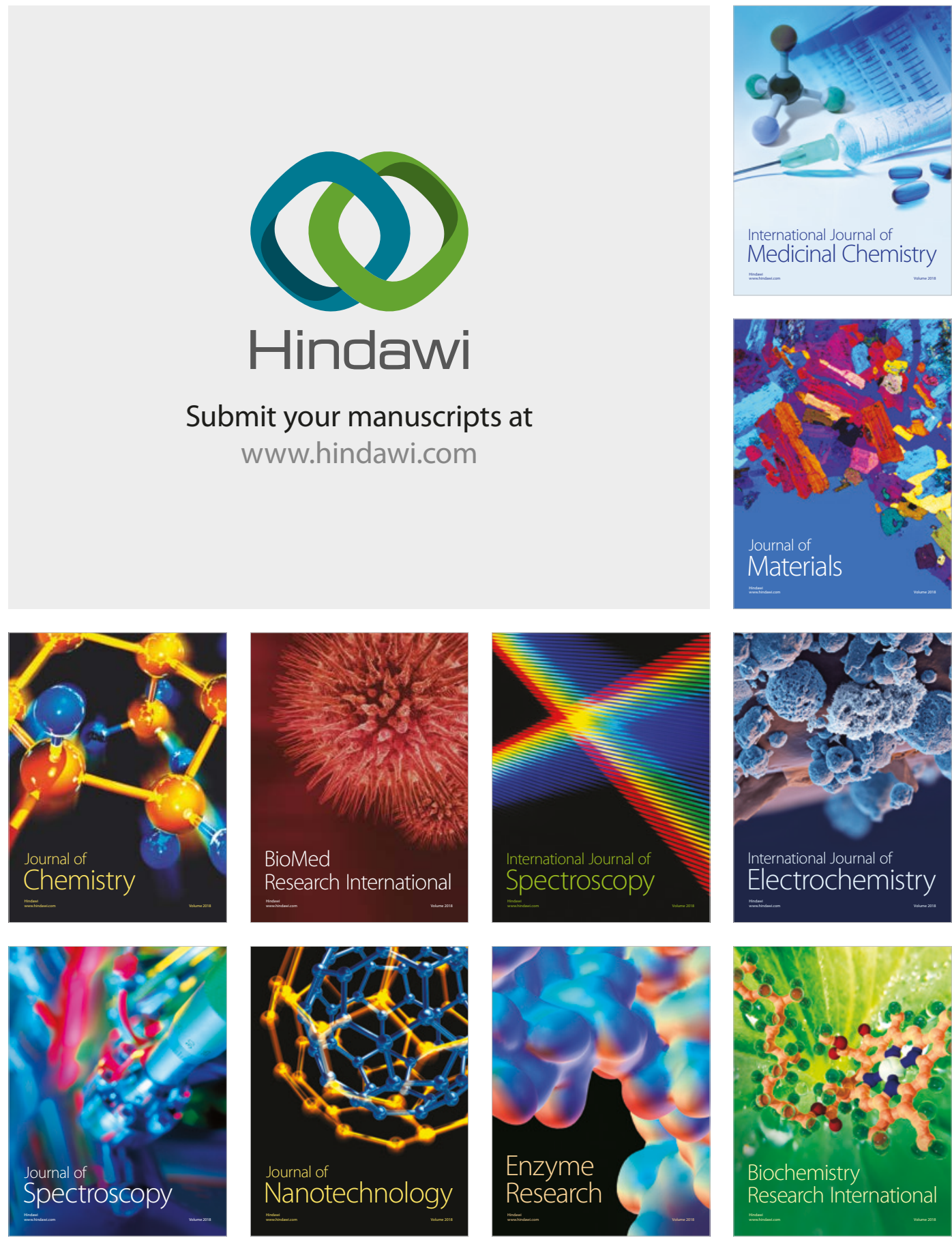
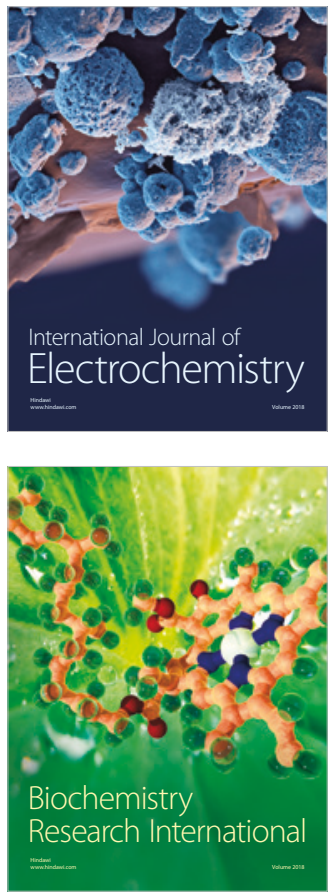